\title{
Spiral Deposition with Alternating Indium Composition in Growing an InGaN Nanoneedle with the Vapor-Liquid-Solid Growth Mode
}

\author{
Wen-Ming Chang, Che-Hao Liao, Chih-Yen Chen, Chieh Hsieh, Tsung-Yi Tang, \\ Yean-Woei Kiang, and C. C. Yang \\ Department of Electrical Engineering, Institute of Photonics and Optoelectronics, National Taiwan University, \\ No. 1 Roosevelt Road, Section 4, Taipei 10617, Taiwan \\ Correspondence should be addressed to C. C. Yang, ccy@cc.ee.ntu.edu.tw
}

Received 16 March 2012; Revised 18 June 2012; Accepted 5 July 2012

Academic Editor: Marinella Striccoli

Copyright (C) 2012 Wen-Ming Chang et al. This is an open access article distributed under the Creative Commons Attribution License, which permits unrestricted use, distribution, and reproduction in any medium, provided the original work is properly cited.

The spiral deposition of InGaN with a quasiperiodical distribution of indium content along the growth direction for forming InGaN nanoneedles (NNs) with the vapor-liquid-solid (VLS) growth mode is demonstrated. The VLS growth is implemented by using $\mathrm{Au}$ nanoparticles (NPs) as the catalyst in metalorganic chemical vapor deposition. The Au NPs on a GaN template are generated through pulsed laser irradiation. The observation of spiral deposition is based on the analyses of the scanning results in the high angle annular dark field and energy dispersive X-ray measurements of transmission electron microscopy. In the measurements, the composition variations along and perpendicular to the growth direction (the $c$-axis) are illustrated. The alternating indium content along the growth direction is attributed to a quasiperiodically pulsed behavior of indium supersaturation process in the melted Au NP at the top of an InGaN NN. The spiral deposition of InGaN is due to the formation of an $\mathrm{NN}$ at the location of an Au NP with a screw-type dislocation beneath in the GaN template, at which the growth of a quasi-one-dimensional structure can be easily initiated.

\section{Introduction}

Quasi-one-dimensional (1D) semiconductor structures have attracted much attention for their potential applications to efficient optoelectronics devices, including light-emitting diode and solar cell. Such a structure has the advantages of low dislocation density, lateral strain relaxation, enhanced light scattering, and larger light/matter interaction surface. In the area of wide-band-gap nitride, self-organized GaN nanorods (NRs) (or called nanowires or nanocolumns) have been widely grown on sapphire and silicon substrates with molecular beam epitaxy (MBE) and metalorganic chemical vapor deposition (MOCVD). With this growth method, GaN NRs can be formed from certain nucleation centers in an AlN or $\mathrm{SiN}_{x}$ buffer layer. In this situation, the planar locations of NRs and their cross-sectional dimensions and heights are randomly distributed $[1,2]$. Self-organized GaN
NRs can also be formed based on the vapor-liquid-solid (VLS) growth mode with the self-catalyst or an extrinsic catalyst (a metal nanoparticle (NP)) [3, 4]. In this growth mode, either the self-catalyst or extrinsic catalyst is used for transferring vapor elements into crystalline structure through the catalytic metal droplet [5-9]. In other words, the melted metal absorbs composition elements to reach a supersaturation condition, under which the composition elements are precipitated on the seeding semiconductor beneath the metal droplet. With this approach, quasi-1D semiconductor structures can be formed following the trace of the metal droplet. In growing GaN NRs, gallium can be used as the self-catalyst in the VLS mode. Besides selforganized growth, selected or patterned growths of $\mathrm{GaN}$ NRs have also been implemented with $\operatorname{MBE}[10,11]$ and MOCVD $[9,12,13]$. In such a regularly patterned growth, vertical NRs of uniform size and height distributions can be 
obtained. With MOCVD growth, the pulsed growth mode, in which the gallium and nitrogen sources are switched on and off alternatively, is usually used for implementing the self-catalyst VLS growth [9, 12, 13]. Besides GaN NRs, In GaN/GaN quantumwell NRs have also been grown for lightemitting applications [14-18].

Because of the large lattice mismatch (11\%) between $\mathrm{GaN}$ and $\mathrm{InN}$, phase separation can occur in InGaN leading to the formation of indium droplets when the incorporated indium content is high [19]. This phenomenon hinters the growth of a high-quality InGaN thin film on a GaN template unless the film is thinner than the critical thickness, which is in the range of a few tens nanometer when the indium content is larger than 20\% [20]. Beyond the critical thickness, the compressive strain in the InGaN thin film from the GaN layer is relaxed. In this situation, to release the strain energy in the InGaN film, phase separation is induced. Therefore, it becomes difficult to grow an InGaN film of a high-indium content. However, in a quasi-1D structure, because the strain can be relaxed in the lateral dimension such that the strain energy can be released to avoid the phase separation behavior, the growth of a high-indium InGaN compound becomes feasible. Also, due to lateral strain relaxation, indium incorporation can be enhanced. The growth of an InGaN NR structure can lead to the formation of high-indium InGaN compounds for the application of nitride-based solar cell. In particular, if the MOCVD growth of InGaN NR structures can be implemented, the industrial development of nitride-based solar cell will become more attractive. Although the growth of an InGaN NR is quite useful, so far, the report on such an accomplishment is still quite rare $[21,22]$, indicating the challenge of this task. Between the self- and extrinsic-catalyst VLS growth modes, because of the ternary composition of InGaN, it is complicated to implement the self-catalyst growth of InGaN. The extrinsiccatalyst VLS mode is an attractive method for growing an InGaN quasi-1D structure. For such a growth process, $\mathrm{Au}$ NPs have been used as the catalyst. Various nanolithography techniques can be used for forming the metal NPs. Also, a lithography-free approach for fabricating densely distributed Au NPs has been demonstrated by irradiating an Au thin film with a high-power laser [23]. It has been reported that a quasi-1D structure can be more easily formed from a nanoscale structure on the template surface [24]. Also, the growth of a quasi-1D structure can be driven or controlled by a screw-type threading dislocation [25-28]. Therefore, a nitride NR can be more easily formed at the location of a V-shaped pit, which usually corresponds to a threading dislocation beneath in the $\mathrm{GaN}$ template. In most $\mathrm{GaN}$ templates grown on sapphire substrate, a large fraction of dislocations belongs to the screw type. Because a dense distribution of metal NPs on the template surface implies a higher probability of locating metal NPs at V-shaped pits, with the metal NP distribution formed through laser irradiation, a higher surface density of nitride NR can be obtained.

In this paper, the spiral deposition behaviors of InGaN with alternating indium composition in growing InGaN nanoneedles (NNs) with the VLS mode are reported. Au NPs formed with laser irradiation onto an $\mathrm{Au}$ thin film

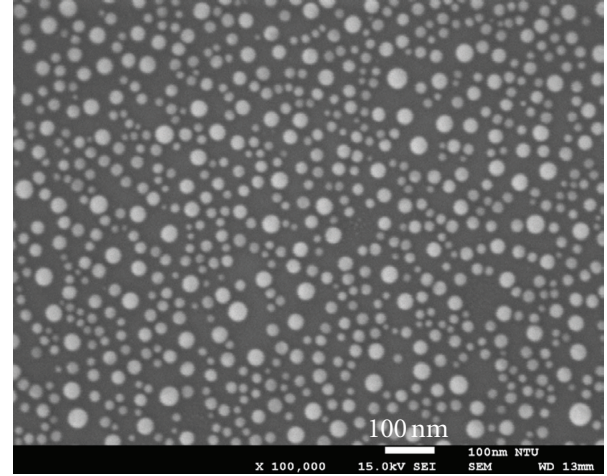

Figure 1: A plan-view SEM image of Au NPs on a GaN template fabricated by laser irradiation.

on a GaN template are used as the catalyst in the VLS growth with MOCVD [23]. From the transmission electron microscopy (TEM) studies, including high angle annular dark field (HAADF) and energy dispersive X-ray (EDX) measurements, we observe the alternating "antisymmetric" distribution of indium content with respect to a vertical axis along the $c$-direction near the center of an InGaN NN. Also, along the growth direction, indium content varies quasiperiodically. It is deduced that the VLS growth follows a spiral deposition pattern of InGaN with a quasiperiodical variation of indium composition in a nanometer scale. In Section 2 of this paper, the InGaN NN growth conditions and the basic characterization results are described. The results of TEM, including HAADF and EDX data, are analyzed and presented in Section 3. Then, discussions are made in Section 4. Finally, conclusions are drawn in Section 5.

\section{InGaN Nanoneedle Growth Conditions and Basic Characterization Results}

The sphere-like Au NPs for serving as the catalyst are prepared on a $2 \mu \mathrm{m} \mathrm{GaN}$ template, which is grown at $1000^{\circ} \mathrm{C}$ on $c$-plane sapphire substrate with MOCVD. They are formed by first depositing an Au thin film of $7.5 \mathrm{~nm}$ in thickness and then irradiating the $\mathrm{Au}$ thin film with the fourth harmonic Q-switched Nd:YAG laser. After the irradiation of five laser pulses of $20 \mathrm{~mJ} / \mathrm{cm}^{2}$ in energy density, sphere-like Au NPs of $75 \mathrm{~nm}$ in average diameter and $2.78 \times 10^{9} \mathrm{~cm}^{-2}$ in surface particle density are fabricated. A plan-view scanning electron microscopy (SEM) image of the Au NPs on GaN is shown in Figure 1. The GaN template with Au NPs is used for GaN and InGaN overgrowth in an MOCVD reactor. Figure 2 shows a cross-sectional SEM image of the GaN overgrowth sample with $\mathrm{GaN}$ deposited at $700^{\circ} \mathrm{C}$ for $30 \mathrm{~min}$. Here, one can see that the $\mathrm{Au}$ NPs are embedded in GaN and no quasi$1 \mathrm{D}$ structure is formed. In other words, $\mathrm{Ga}$ atoms are not effectively absorbed by melted Au NPs for VLS growth. It is noted that the $\mathrm{Au}$ NPs are expected to be melting at the temperature of $700^{\circ} \mathrm{C}$ even though it is significantly lower than the melting point of bulk Au at $1064^{\circ} \mathrm{C}$.

Then, InGaN overgrowth is performed on another GaN template with similar $\mathrm{Au}$ NPs also at $700^{\circ} \mathrm{C}$ for $60 \mathrm{~min}$ after 


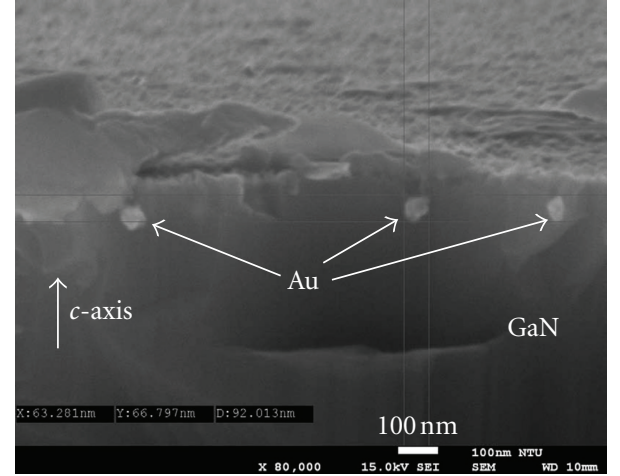

FIGURE 2: A cross-sectional SEM image of a GaN layer with embedded Au NPs.

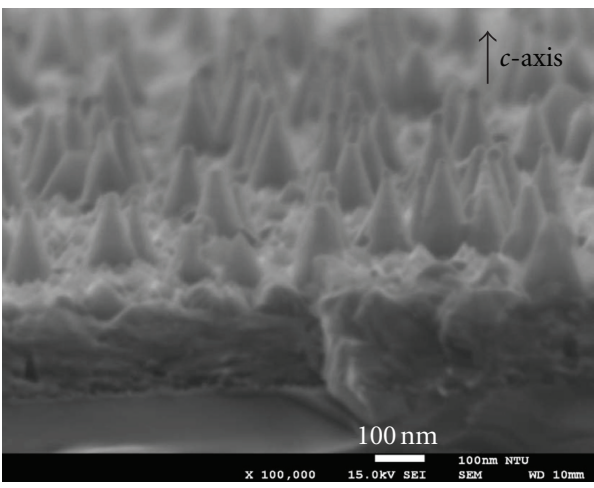

Figure 3: A tilted SEM image of the grown InGaN NNs.

a stage of $\mathrm{GaN}$ growth at $600^{\circ} \mathrm{C}$ for $10 \mathrm{~min}$. This stage of $\mathrm{GaN}$ growth is needed for filling up the space between $\mathrm{Au}$ NPs before InGaN growth. Without this stage, no quasi$1 \mathrm{D}$ InGaN structure can be fabricated. Figure 3 shows a tilted SEM image of the grown InGaN NNs. At the tips of some InGaN NNs, the residual Au NPs (dark spots) can still be seen. The bases of some of those NNs are larger than $100 \mathrm{~nm}$ in size, which is larger than the average dimension of the formed Au NPs. Neighboring Au NPs on the GaN template can be combined to form a larger melted Au droplet for serving as the catalyst. Au atoms may be mixed into precipitated InGaN during the catalytic growth such that the size of an Au droplet becomes smaller and the cross-section of an InGaN NR also becomes smaller along the growth in the $c$-direction to form the needle geometry.

Figure 4 demonstrates a cross-sectional TEM HAADF image showing three InGaN NNs with the residual Au NPs being removed (to show the flat tops). The heights of the InGaN NNs are around $170 \mathrm{~nm}$. Their bases are in the range between 120 and $130 \mathrm{~nm}$. A Philips Tecnai F20 G2 FEITEM system is used for HAADX and EDX observations with the size of electron beam at $1 \mathrm{~nm}$. Also, a Bede 1 highresolution X-ray diffraction (XRD) facility is used for XRD evaluation of the InGaN NN sample. From the result of reciprocal space mapping (RSM), as shown in Figure 5, the In $\mathrm{GaN}$ is fully strain relaxed. Here, the vertical and slant

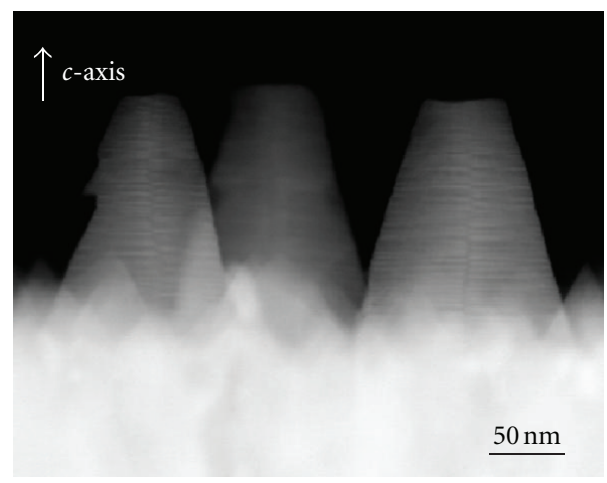

FIgURE 4: A cross-sectional TEM HAADF image showing three InGaN NNs.

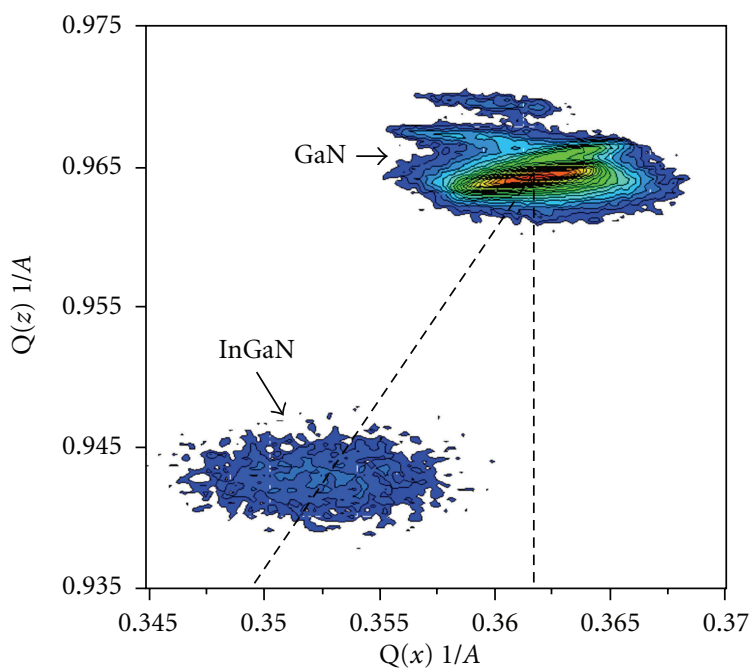

FIGURE 5: RSM image of the InGaN NN sample with the vertical and slant dashed lines corresponding to the fully strained and fully strain-relaxed InGaN conditions, respectively.

dashed lines correspond to the fully strained and fully strainrelaxed conditions, respectively. Figure 6 shows the XRD $\omega$ $2 \theta$-scan intensity distribution of the InGaN NN sample. The InGaN peak here corresponds to an average indium content of $22 \%$. Figure 7 shows the photoluminescence (PL) spectra at various temperatures from 10 through $300 \mathrm{~K}$. The data are obtained by exciting the sample with a $\mathrm{HeCd}$ laser at $325 \mathrm{~nm}$ in wavelength and $5 \mathrm{~mW}$ in power from the top surface. Here, two major emission features at $\sim 530$ and $\sim 600 \mathrm{~nm}$ can be seen. When the sample temperature is lower than $120 \mathrm{~K}$, the high-energy feature dominates. Above $\sim 120 \mathrm{~K}$, the low-energy feature becomes stronger and the high-energy peak diminishes. Also, the low-energy peak red shifts with increasing temperature. The 530 and $600 \mathrm{~nm}$ features roughly correspond to the indium contents of 16 and $23 \%$, respectively, which are located within the XRD hump of InGaN in Figure 6. At a low temperature, the PL emission mainly originates from the low-indium portion of higher crystal quality (and possibly a relatively larger volume) in an NN. As temperature increases, thermalized carriers flow into 


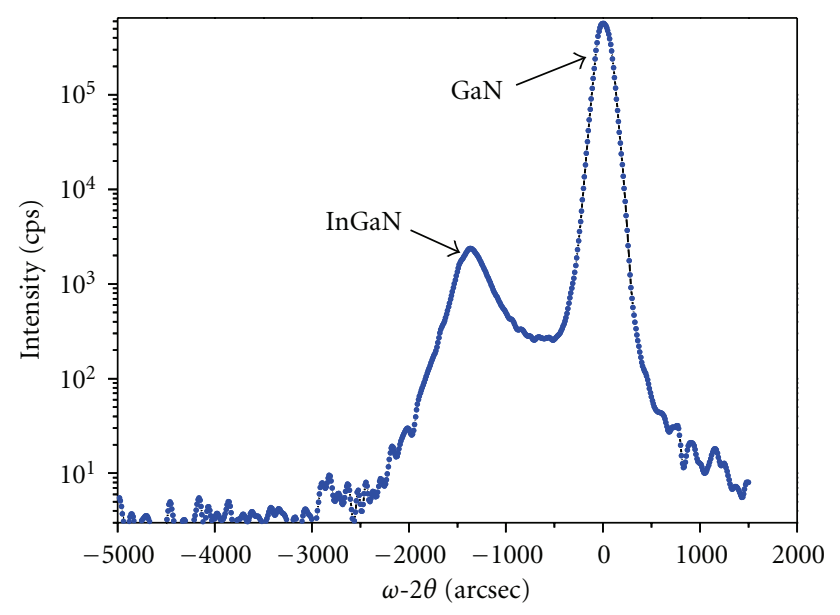

Figure 6: XRD $\omega-2 \theta$ scan pattern of the InGaN NN sample.

the high-indium portion of lower potential for recombination and emission. It is speculated that a potential barrier exists between the high- and low-indium portions such that certain thermal energy is required for carriers to flow across the boundary. From the PL measurement, one can see the broad-range distribution of indium composition in an InGaN NN. Roughly speaking, two indium composition levels exist in such an InGaN nanostructure.

\section{Analyses of Transmission Electron Microscopy Images}

Figure 8 shows the close-up HAADF image of an InGaN NN. The circular bright spots in the background correspond to the residual $\mathrm{Au}$ NPs. In the NN, one can clearly observe a vertical axis around the center of the $\mathrm{NN}$ (indicated by the two arrows in the $\mathrm{NN}$ region). Also, the bright and dark contrast distribution shows a quasiperiodical pattern along the vertical direction (the $c$-axis) and a roughly "antisymmetric" pattern along the horizontal direction with respect to the aforementioned vertical axis. In other words, if the image is brighter on the right-hand side at a certain height of the NN, it is generally darker on the left-hand side at the same height of the NN. Also, it becomes darker on the same side in the next half cycle of the quasiperiod when moving along the vertical axis. It is noted that in a HAADF image, a brighter region corresponds to higher indium content. Therefore, it is deduced that the InGaN growth follows a spiral deposition pattern with a quasiperiodical distribution of indium content along the growth direction [29-34]. Figure 9 shows the top-view SEM image of an InGaN NN. Here, we can see the clockwise spiral structure of the NN, which is formed when its cross-sectional dimension is reduced along the $c$-axis, as indicated by the curved arrows in Figure 9.

To further demonstrate the spiral growth structure, the vertical and horizontal distributions of EDX signal and HAADF intensity roughly along the marked vertical and horizontal dashed lines in Figure 8 (red and blue dashed lines for EDX and HAADF, resp.) are analyzed. Figure 10

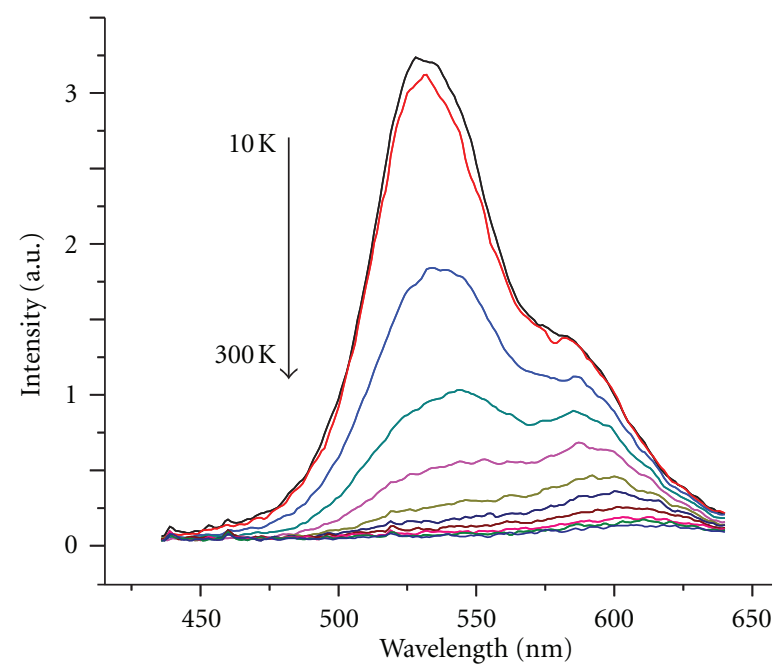

Figure 7: PL spectra of the InGaN NN sample at various temperatures.

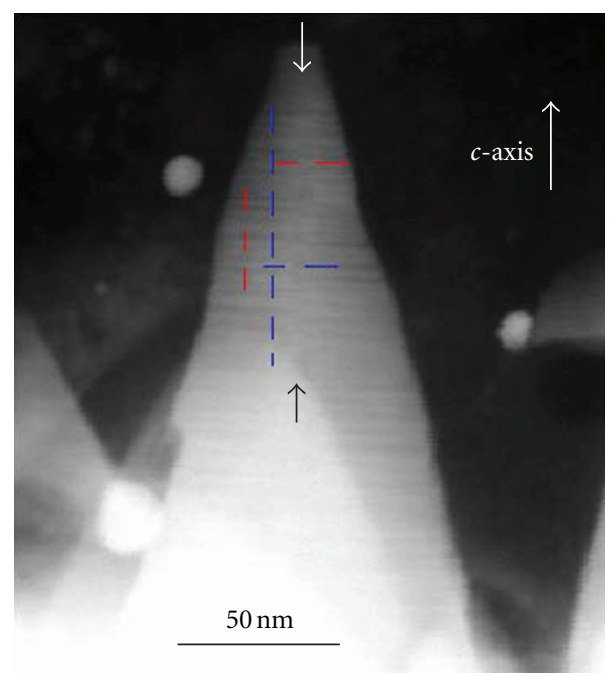

FIGURE 8: HAADF close-up image of an InGaN NN. The vertical growth axis is indicated by the two short vertical arrows. Two horizontal and two vertical dashed lines are drawn to show the rough locations of HAADF and EDX scanning for analysis.

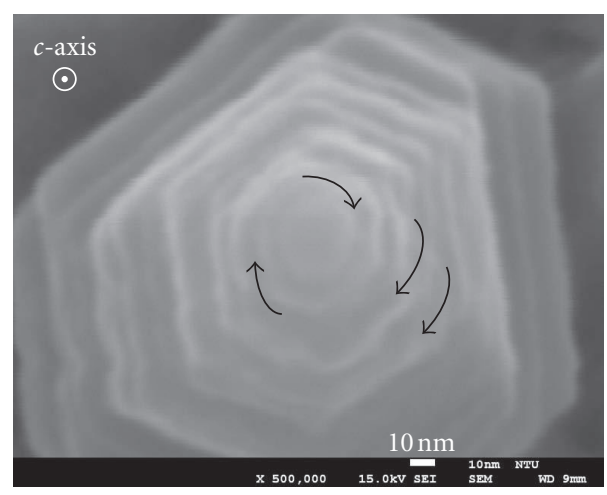

Figure 9: Top-view SEM image of an InGaN NN to show the spiral structure. 


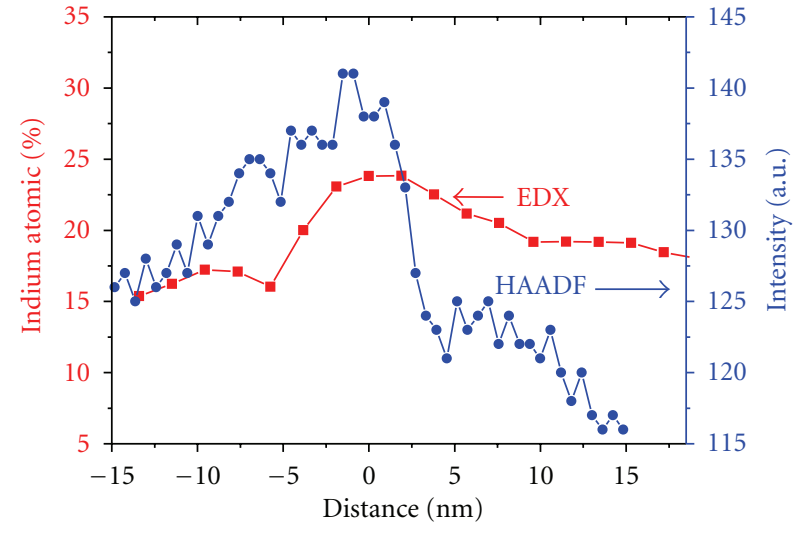

FIGURE 10: Horizontal EDX (the left ordinate for indium atomic $\%$ ) and HAADF (the right ordinate for intensity) data distributions along the horizontal dashed lines shown in Figure 8.

shows the EDX (the left ordinate for indium atomic \%) and HAADF (the right ordinate for intensity) data distributions along the horizontal dashed lines (at different heights) in Figure 8. The spatial resolution of EDX scanning is around $1 \mathrm{~nm}$. That of HAADF is expected to be higher. In Figure 10, generally two different levels can be seen in either indium atomic \% of EDX or HAADF intensity between the positive and negative coordinates. Here, the zero points of the horizontal coordinates for the EDX and HAADF data are roughly assigned to show the "antisymmetric" patterns. Around the vertical axis (at 0 distance here), either HAADF intensity or EDX indium atomic \% shows a local maximum.

Figure 11 shows the results of HAADF and EDX scanning, similar to Figure 10, along the vertical direction. The generally decreasing trend of HAADF intensity along the $c$ axis is caused by imperfect imaging operation and cannot be interpreted as a decreasing trend of indium content. To see the nanoscale variation of indium composition along the $c$-axis, we perform the Fourier transforms of the spatial signal variations in Figure 11 to give the normalized spatialfrequency spectra in Figure 12. In this figure, although one cannot observe any dominating spatial-frequency component within the concerned range $\left(>0.1 \mathrm{~nm}^{-1}\right)$ in either HAADF or EDX scanning result, a few consistent peaks between the two sets of data can be identified, including those at 0.22 and $0.3 \mathrm{~nm}^{-1}$. These two spatial-frequency features correspond to 4.55 and $3.33 \mathrm{~nm}$ in period in a quasiperiodical variation of indium content. They represent the two major components in indium content variation.

\section{Discussions}

Based on the "antisymmetric" HAADF and EDX data distributions with respect to the vertical axis, one can propose a mechanism of spiral deposition of $\mathrm{InGaN}$ atoms with varying indium composition. Because a TEM image shows the integration of atomic distribution along the electron beam direction, the projection of such an integration of a spiral indium distribution can lead to the alternating HAADF contrast along the growth axis with an "antisymmetric" distribution

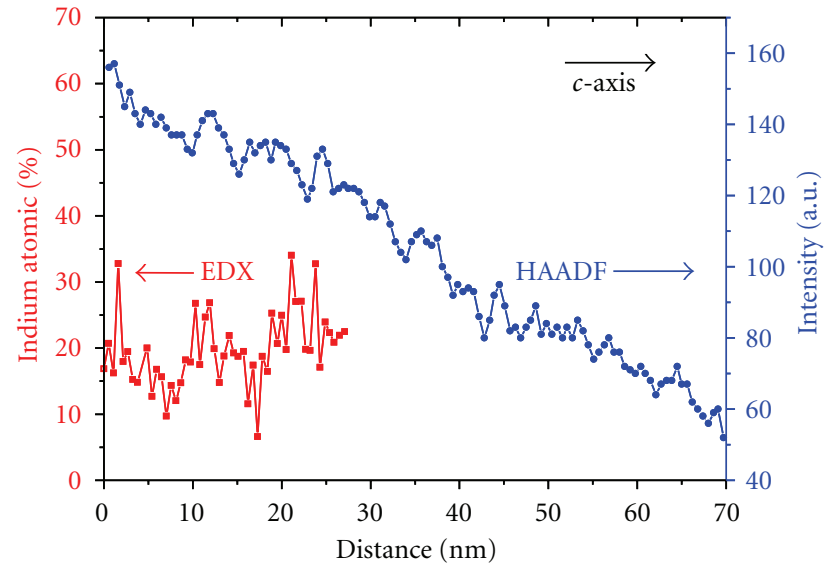

FIGURE 11: Vertical EDX (the left ordinate for indium atomic \%) and HAADF (the right ordinate for intensity) data distributions along the vertical dashed lines shown in Figure 8.

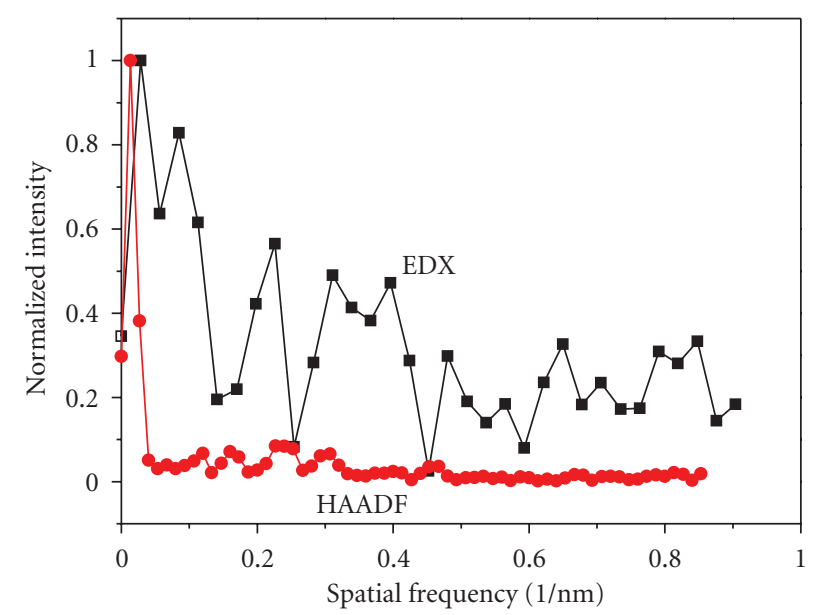

FIgURE 12: Normalized spatial-frequency spectra of the HAADF and EDX data in Figure 11.

with respect to the axis of the InGaN NN. Also, because of the thickest integration around the axis, a maximum of projected indium content can be observed around this axis. Such results can be visualized by looking at a mechanical spring in a direction perpendicular to its axis. Spiral growths in various materials with the VLS mode have been reported [29-34]. Based on the reported theories and simulations, its growth mechanism has been attributed to the formation of a screw dislocation in the earlystage of the growth [29, 30, 32, 34]. A screw dislocation in the GaN template, which can be formed in a GaN film on sapphire substrate with a high surface density (up to $10^{10} \mathrm{~cm}^{-2}$ ), can be easily covered by an Au NP in our experiment and results in the spiral deposition of an InGaN NN.

The alternating indium composition can be attributed to a noncritical supersaturation process during the VLS growth. In other words, there is a finite range of indium content for reaching the supersaturation and precipitation conditions in the Au droplet. From the results of $\mathrm{GaN}$ overgrowth 
(see Figure 2), in which Au NPs are buried in GaN and no quasi-1D structure is formed, it is reasonable to assume that $\mathrm{Ga}$ incorporation into the melted $\mathrm{Au}$ droplet is less effective than its direct deposition. Therefore, the grown InGaN composition is controlled by the indium incorporation into the melted $\mathrm{Au}$ droplet at the needle top. During indium precipitation to form InGaN, the major part of $\mathrm{Ga}$ atoms is supplied directly from the vapor phase. Such a Ga atom supply is supposed to be quite stable. Once the supersaturation condition is reached, $\mathrm{InGaN}$ is deposited through precipitation to form a high-indium layer of a couple $\mathrm{nm}$ in thickness. This supersaturation and hence precipitation condition can be maintained for a certain period even though the indium content in the $\mathrm{Au}$ droplet is slightly reduced. Under such a condition, an InGaN layer of a relatively lower indium content is grown until the next supersaturation stage is reached and another higher-indium InGaN layer is to be deposited. The mechanism for generating such a pulsed supersaturation process is still unclear and deserves further investigation. It is noted that with the random distribution of Au NPs or InGaN NNs on the GaN template and the turbulent ambient vapor in the MOCVD growth chamber, the pulsed supersaturation process cannot follow a perfectly periodical pattern. Hence, the quasiperiodical behavior is observed.

\section{Conclusions}

In summary, the spiral deposition of InGaN with a quasiperiodical distribution of indium content along the growth direction for forming InGaN NNs with the VLS mode was observed. The observation was based on the analyses of the HAADF and EDX scanning results along and perpendicular to the growth direction. The alternating indium content along the growth direction was attributed to a pulsed behavior of the indium supersaturation process in the catalytic Au droplet at the top of an InGaN NN. The spiral deposition of In GaN was due to the formation of an NN at the location of an Au NP with a screw-type dislocation beneath in the GaN template, at which the growth of a quasi-1D structure could be easily initiated.

\section{Acknowledgments}

This research was supported by National Science Council, Taiwan, The Republic of China, under the Grants of NSC 99-2221-E-002-123-MY3, 100-2622-E-002-008-CC2, and 100-2221-E-002-170, by NTU Excellent Research Project (10R80908-B), by Epistar Corporation, and by US Air Force Scientific Research Office under the Contract of AOARD-114114 .

\section{References}

[1] L. W. Tu, C. L. Hsiao, T. W. Chi, I. Lo, and K. Y. Hsieh, "Selfassembled vertical GaN nanorods grown by molecular-beam epitaxy," Applied Physics Letters, vol. 82, no. 10, pp. 1601-1603, 2003.

[2] C. Chèze, L. Geelhaar, B. Jenichen, and H. Riechert, "Different growth rates for catalyst-induced and self-induced GaN nanowires," Applied Physics Letters, vol. 97, no. 15, Article ID 153105, 2010.

[3] T. Song, W. I. Park, and U. Paik, "Epitaxial growth of onedimensional GaN nanostructures with enhanced near-band edge emission by chemical vapor deposition," Applied Physics Letters, vol. 96, no. 1, Article ID 011105, 2010.

[4] X. J. Chen, G. Perillat-Merceroz, D. Sam-Giao, C. Durand, and J. Eymery, "Homoepitaxial growth of catalyst-free $\mathrm{GaN}$ wires on N-polar substrates," Applied Physics Letters, vol. 97, no. 15, Article ID 151909, 2010.

[5] R. S. Wagner and W. C. Ellis, "Vapor-liquid-solid mechanism of single crystal growth," Applied Physics Letters, vol. 4, no. 5, pp. 89-90, 1964.

[6] J. V. Milewski, F. D. Gac, J. J. Petrovic, and S. R. Skaggs, "Growth of beta-silicon carbide whiskers by the VLS process," Journal of Materials Science, vol. 20, no. 4, pp. 1160-1166, 1985.

[7] H. L. Qiu, C. B. Cao, X. Xiang, Y. H. Zhang, J. Li, and H. S. Zhu, "Large scale tapered GaN rods grown by chemical vapour deposition," Journal of Crystal Growth, vol. 290, pp. 1-5, 2006.

[8] T. Kuykendall, P. J. Pauzauskie, Y. Zhang et al., "Crystallographic alignment of high-density gallium nitride nanowire arrays," Nature Materials, vol. 3, no. 8, pp. 524-528, 2004.

[9] T. Y. Tang, W. Y. Shiao, C. H. Lin et al., "Coalescence overgrowth of GaN nanocolumns on sapphire with patterned metal organic vapor phase epitaxy," Journal of Applied Physics, vol. 105, no. 2, Article ID 023501, 2009.

[10] Y. Kawakami, S. Suzuki, A. Kaneta, M. Funato, A. Kikuchi, and K. Kishino, "Origin of high oscillator strength in greenemitting InGaN/GaN nanocolumns," Applied Physics Letters, vol. 89, no. 16, Article ID 163124, 2006.

[11] K. Kishino, H. Sekiguchi, and A. Kikuchi, "Improved Ti-mask selective-area growth (SAG) by rf-plasma-assisted molecular beam epitaxy demonstrating extremely uniform GaN nanocolumn arrays," Journal of Crystal Growth, vol. 311, no. 7, pp. 2063-2068, 2009.

[12] S. D. Hersee, X. Sun, and X. Wang, "The controlled growth of GaN nanowires," Nano Letters, vol. 6, no. 8, pp. 1808-1811, 2006.

[13] Y. S. Chen, W. Y. Shiao, T. Y. Tang et al., "Threading dislocation evolution in patterned GaN nanocolumn growth and coalescence overgrowth," Applied Physics Letters, vol. 106, Article ID 023521, 2009.

[14] W. Gao, M. Zhang, A. Banerjee, and P. Bhattacharya, "Catalyst-free InGaN/GaN nanowire light emitting diodes grown on (001) silicon by molecular beam epitaxy," Nano Letters, vol. 10, no. 9, pp. 3355-3359, 2010.

[15] H. Sekiguchi, K. Kishino, and A. Kikuchi, "Emission color control from blue to red with nanocolumn diameter of InGaN/GaN nanocolumn arrays grown on same substrate," Applied Physics Letters, vol. 96, Article ID 231104, 2010.

[16] H. W. Lin, Y. J. Lu, H. Y. Chen, H. M. Lee, and S. Gwo, "InGaN/GaN nanorod array white light-emitting diode," Applied Physics Letters, vol. 97, Article ID 073101, 2010.

[17] Y. L. Chang, J. L. Wang, F. Li, and Z. Mi, "High efficiency green, yellow, and amber emission from InGaN/GaN dot-in-a-wire heterostructures on Si(111)," Applied Physics Letters, vol. 96, Article ID 013106, 2010.

[18] H. P. T. Nguyen, S. Zhang, K. Cui et al., "P-type modulation doped InGaN/GaN dot-in-a-wire white-light-emitting diodes monolithically grown on Si(111)," Nano Letters, vol. 11, no. 5, pp. 1919-1924, 2011.

[19] I. H. Ho and G. B. Stringfellow, "Solid phase immiscibility in GaInN,” Applied Physics Letters, vol. 69, Article ID 2701, 1996. 
[20] C. A. Parker, J. C. Roberts, S. M. Bedair, M. J. Reed, S. X. Liu, and N. A. El-Masry, "Determination of the critical layer thickness in the InGaN/GaN heterostructures," Applied Physics Letters, vol. 75, no. 18, pp. 2776-2778, 1999.

[21] H.-M. Kim, W. C. Lee, T. W. Kang, K. S. Chung, C. S. Yoon, and C. K. Kim, "InGaN nanorods grown on (1 111$)$ silicon substrate by hydride vapor phase epitaxy," Chemical Physics Letters, vol. 380, no. 1-2, pp. 181-184, 2003.

[22] K. M. Wu, Y. Pan, and C. Liu, "InGaN nanorod arrays grown by molecular beam epitaxy: Growth mechanism structural and optical properties," Applied Surface Science, vol. 255, no. 13-14, pp. 6705-6709, 2009.

[23] C. Y. Chen, J. Y. Wang, F. J. Tsai, Y. C. Lu, Y. W. Kiang, and C. C. Yang, "Fabrication of sphere-like Au nanoparticles on substrate with laser irradiation and their polarized localized surface plasmon behaviors," Optics Express, vol. 17, no. 16, pp. 14186-14198, 2009.

[24] R. Koester, J. S. Hwang, C. Durand, D. Le Si Dang, and J. Eymery, "Self-assembled growth of catalyst-free GaN wires by metal-organic vapour phase epitaxy," Nanotechnology, vol. 21, no. 1, Article ID 015602, 2010.

[25] D. Cherns, L. Meshi, I. Griffiths et al., "Defect-controlled growth of GaN nanorods on (0001)sapphire by molecular beam epitaxy," Applied Physics Letters, vol. 93, no. 11, Article ID 111911, 2008.

[26] S. A. Morin, M. J. Bierman, J. Tong, and S. Jin, "Mechanism and kinetics of spontaneous nanotube growth driven by screw dislocations," Science, vol. 328, no. 5977, pp. 476-480, 2010.

[27] W. Ye, B. Paliwal, W. H. Goh, M. Cherkaoui, and A. Ougazzaden, "Finite element modeling of dislocation in solids and its applications to the analysis of GaN nanostructures," Computational Materials Science, vol. 58, pp. 154-161, 2012.

[28] S. Jin, M. J. Bierman, and S. A. Morin, "A new twist on nanowire formation: Screw-dislocation-driven growth of nanowires and nanotubes," Journal of Physical Chemistry Letters, vol. 1, no. 9, pp. 1472-1480, 2010.

[29] S. Harris and P. Smilauer, "Analytical solution of generalized Burton-Cabrera-Frank equations for growth and post-growth equilibration on vicinal surfaces," Physical Review B, vol. 50, no. 11, pp. 7952-7961, 1994.

[30] A. Karma and M. Plapp, "Spiral surface growth without desorption," Physical Review Letters, vol. 81, no. 20, pp. 4444 4447, 1998.

[31] A. Kimura, N. Futagawa, A. Usui, and M. Mizuta, "Supersaturation-dependent step-behavior of InGaN grown by metal organic vapor phase epitaxy," Journal of Crystal Growth, vol. 229, no. 1, pp. 53-57, 2001.

[32] P. M. Martins and F. Rocha, "Characterization of crystal growth using a spiral nucleation model," Surface Science, vol. 601, no. 16, pp. 3400-3408, 2007.

[33] N. Wang, Y. Cai, and R. Q. Zhang, "Growth of nanowires," Materials Science and Engineering R: Reports, vol. 60, no. 1-6, pp. 1-51, 2008.

[34] A. Redinger, O. Ricken, P. Kuhn et al., "Spiral growth and Step edge barriers," Physical Review Letters, vol. 100, no. 3, Article ID 035506, 2008. 

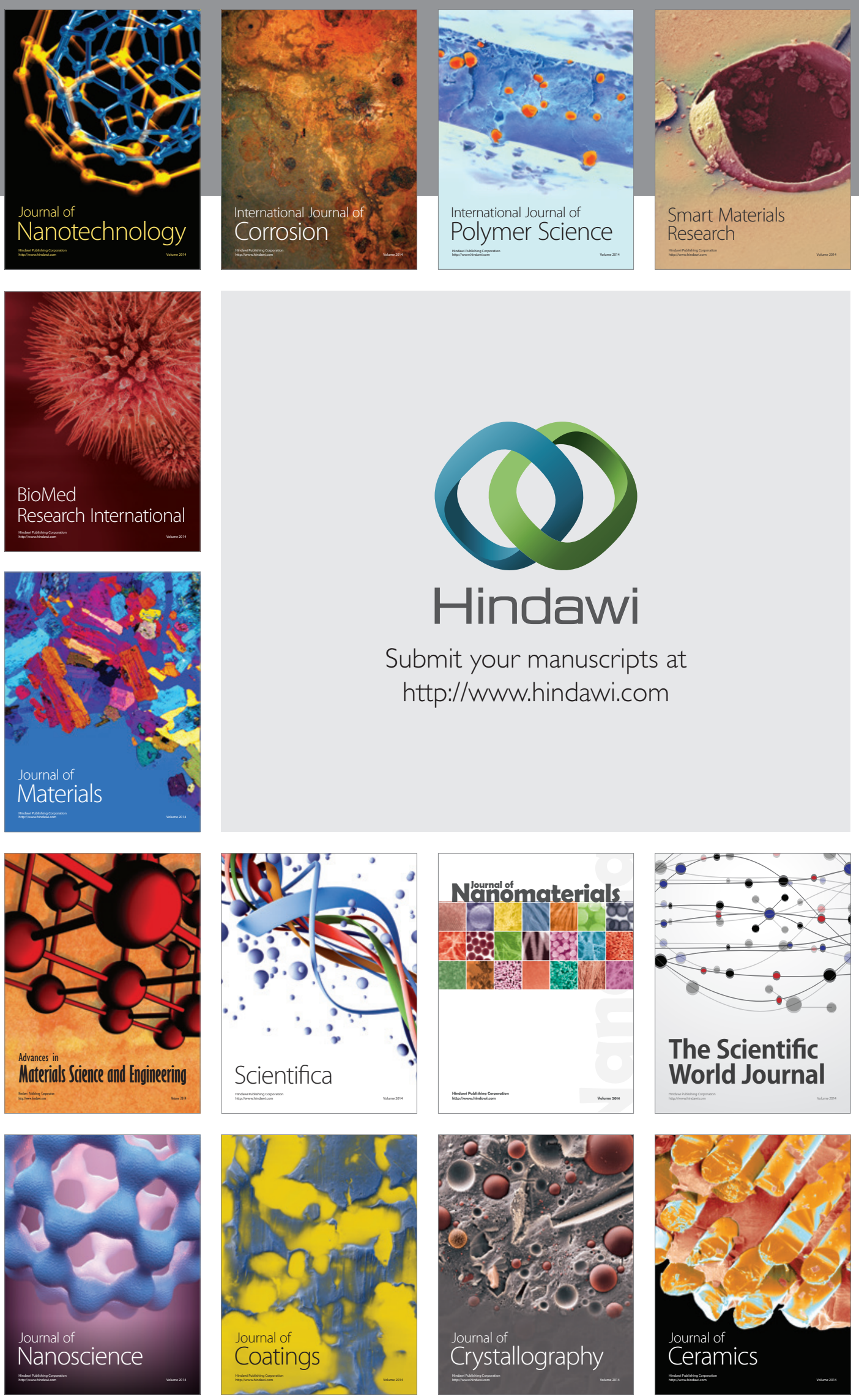

The Scientific World Journal

Submit your manuscripts at

http://www.hindawi.com

\section{World Journal}

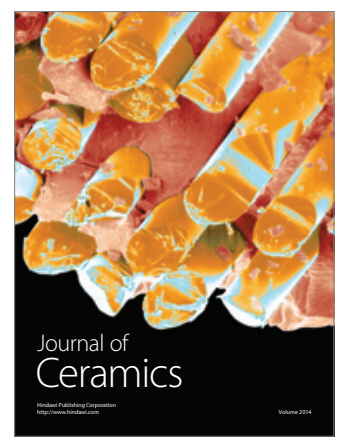

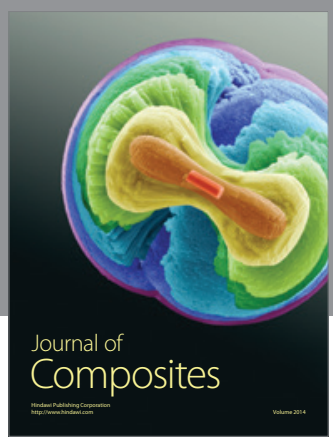
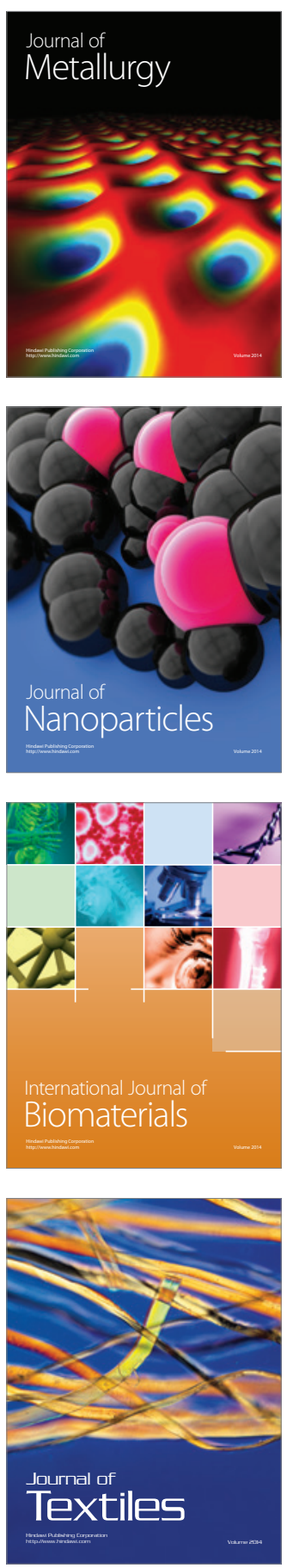